Brit. Heart f., 1968, 30, 309.

\title{
Clinical Features of Endomyocardial Fibrosis of the Right Ventricle
}

\author{
K. SOMERS , D.P. BRENTON, AND N. K. SOOD \\ From the Department of Medicine, Mulago Hospital, and the Department of Physiology, Makerere University \\ Medical School, Kampala, Uganda
}

Endomyocardial fibrosis was first described from Uganda as a pathological entity distinct from other forms of heart disease by Davies (1948). The characteristic lesion of the disorder is a fibrosis of the endocardium and subendocardial tissues of the ventricles, which may involve the papillary muscles and cause valvular incompetence (Fig. 1). The incidence of endomyocardial fibrosis at the Mulago Hospital Cardiac Clinic has been estimated at approximately 12 per cent of all cardiac diseases, excluding hypertensive heart disease (Somers and D'Arbela, 1964). The aetiology of endomyocardial fibrosis is obscure. Van der Geld et al. (1966) have demonstrated a predisposition to auto-immune reactivity to the heart in endomyocardial fibrosis. A recent pathological study of 16 necropsy specimens, using histochemical techniques, suggests that the fibrotic scarring is preceded by changes in the cardiac ground substance and that the initial insult may be upon this structure rather than upon the cells themselves (Connor et al., 1967, 1968).

The early clinical description of endomyocardial fibrosis from Mulago Hospital was by Ball, Williams, and Davies (1954). They described the basic clinical features of 20 cases, proven at necropsy, of which 5 probably had predominant involvement of the right ventricle. Shillingford and Somers (1961) studied 15 patients with endomyocardial fibrosis by right heart catheterization. One-third of them probably had a predominantly right ventricular lesion but all the others had a moderate or severe pulmonary hypertension, suggesting considerable left ventricular disease. The clinical features of the right ventricular disease as it is seen in Nigeria have been reviewed by Abrahams (1962), and a tentative outline for the possible natural history of

\footnotetext{
Received June 1, 1967.

* In receipt of grants from the Medical Research Council, London, and the British Heart Foundation.
}

endomyocardial fibrosis has been offered by Parry and Abrahams (1965). The same authors have published haemodynamic data from studies on 30 patients of whom many had moderate pulmonary hypertension, suggesting that they had biventricular fibrosis (Parry and Abrahams 1963). The relatively frequent occurrence of pericardial effusion in association with endomyocardial fibrosis was reported by Williams, Ball, and Davies (1954), and Abrahams and Parry (1963) published the features of 6 patients with chronic pericardial effusion and right ventricular endomyocardial fibrosis.

The purpose of this present paper is to report the clinical features of 28 patients with right ventricular endomyocardial fibrosis who have been studied in Mulago Hospital. In general our findings agree with those published from Nigeria though they differ in detail.

\section{Patients}

When endomyocardial fibrosis affects only the right ventricle the signs of right-sided heart failure are marked, but clinical and radiological evidence of pulmonary congestion is absent. Left ventricular involvement results in pulmonary congestion and dyspnoea, pulmonary hypertension, and frequently mitral incompetence. The patients reported in this series all had severe involvement of the right ventricle. Eight patients had systolic murmurs audible at the mitral area suggesting the presence of left ventricular involvement but none had marked pulmonary hypertension, and only 2 had dyspnoea with pulmonary congestion. In some of these patients it is possible that the systolic murmur heard at the mitral area was tricuspid in origin.

Two of the patients have died and the diagnosis was confirmed at necropsy. In another 3 the diagnosis was confirmed at exploratory thoracotomy undertaken to exclude constrictive pericarditis. Nineteen others have had cine-angiographic studies 


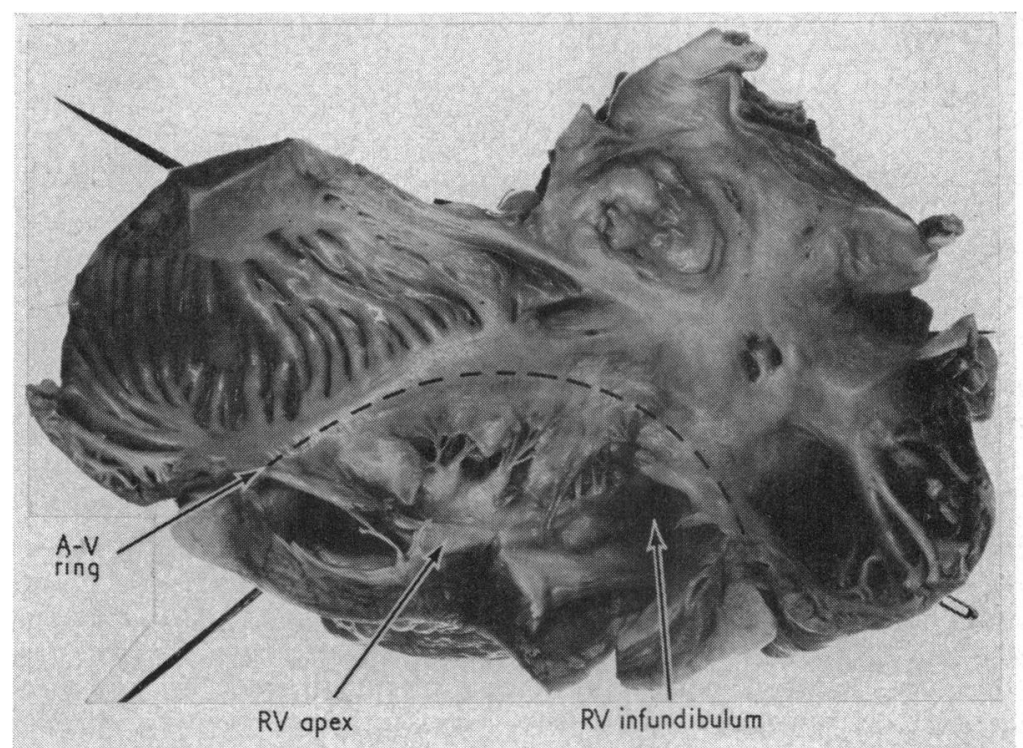

FIG. 1. Necropsy specimen of a heart with endomyocardial fibrosis of the right ventricle (RV). The fibrosis spreads up from the apex to involve the papillary muscles and chordae. The right atrium is large and its wall is hypertrophied. (A-V atrioventricular)

of the right side of the heart, and the appearances were typically those described by Cockshott (1965) in severe right ventricular endomyocardial fibrosis. The clinical features of 2 of the 28 patients have been partly described elsewhere (Shillingford and Somers, 1961: Case 10; W.H.O. Memorandum, 1965).

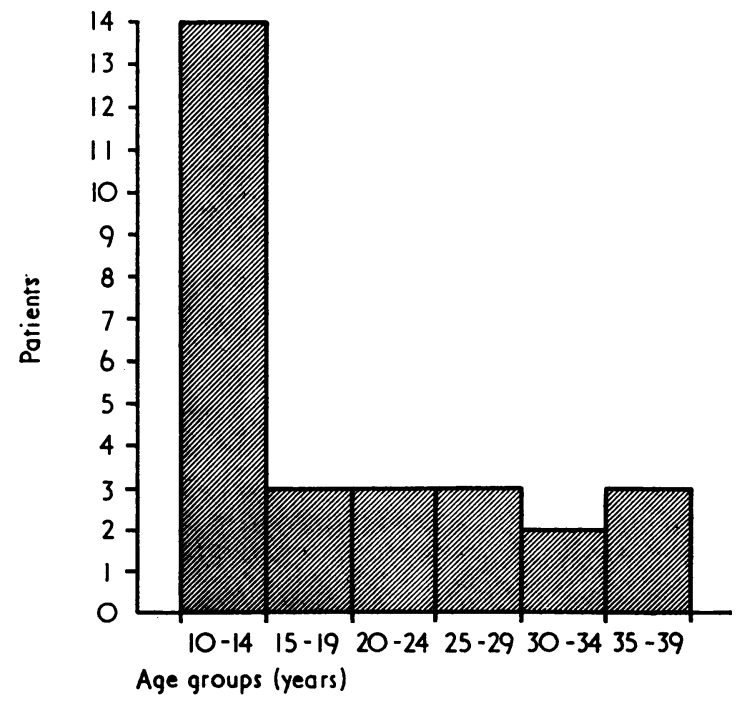

FIG. 2. Age at the onset of symptoms in 28 patients with endomyocardial fibrosis.
Tribe, Sex, and Age. Patients of many different tribes come to Mulago Hospital. People of the Ganda tribe predominate, since this is the local Ugandan tribe. Some of the patients are immigrants from the neighbouring countries of Rwanda, Burundi, Tanzania, Kenya, and the Sudan. Nineteen of the 28 patients reported here were Rwandans; most had lived in Uganda a number of years. Only 4 were Ganda people, and the 5 others were from 5 different Ugandan and Tanzanian tribes. The sex incidence was almost equal: 15 male and 13 female. The distribution of patients according to age at the onset of symptoms is shown in Fig. 2. Many of the patients were under 15 years old when symptoms began, the mean age of onset being 19 years. The mean was higher in females $(23.5$ years) than in males (15 years).

\section{SYMPTOMS}

The time from the onset of symptoms to presentation at Mulago varied from less than a month to several years, the average being 15 months. Most patients presented, therefore, in the chronic stage of the illness. Table I shows the frequency of various symptoms.

The onset of the disease was insidious in most cases, with no clear history of an acute illness at the onset. Most patients presented with abdominal swelling due to ascites and abdominal pain due either to venous engorgement of the liver or 
TABLE I

PRESENTING SYMPTOMS IN 28 PATIENTS WITH ENDOMYOCARDIAL FIBROSIS

\begin{tabular}{l|c}
\hline \multicolumn{1}{c|}{ Symptoms } & $\begin{array}{c}\text { No. of } \\
\text { patients }\end{array}$ \\
\cline { 1 - 2 } Abdominal swelling & 28 \\
Abdominal pain & 21 \\
Ankle swelling & 17 \\
Exertional dyspnoea & 18 \\
Cough & 18 \\
Facial swelling & 17 \\
Palpitations & 14 \\
Fever & 12 \\
Nocturnal dyspnoea & 7 \\
Chest pain & 5 \\
Joint pains & 4 \\
Sore throat & 4 \\
Haemoptysis & 2 \\
\end{tabular}

stretching of the abdominal wall. Patients frequently said that the ascites preceded the oedema of face and ankles. Exertional dyspnoea was usually not due to pulmonary congestion and was frequently relieved by tapping a large tense ascites, and this also relieved nocturnal dyspnoea in some cases. Cough occurred in over half the subjects but was rarely severe and usually productive of only scanty white sputum. Palpitation was commonly noticed on exertion. Descriptions of chest pain were vague and, though praecordial in position, it was quite unlike anginal pain in most respects. Fever was a difficult symptom to evaluate since the term is frequently used by patients in Uganda to mean nothing more than general malaise. Many of the patients admitted to general weakness, loss of energy, and tiredness which are features commonly associated with a low cardiac output. Some considered they were gradually losing weight.

\section{Physical Signs}

Table II shows the incidence of the principal signs outside the heart and lungs.

The triad of raised venous pressure, hepatomegaly, and ascites occurred in all cases. The average of the mean right atrial pressures in the 28 cases was $20 \mathrm{~mm}$. $\mathrm{Hg}$ at cardiac catheterization. Clinically, 13 patients appeared to have a large systolic venous wave suggestive of tricuspid incompetence, and 9 of these had systolic pulsation of the liver. The $y$ descent was nearly always a prominent feature of the venous pulse. Ascites was gross and tense in 22 patients, moderate in 4, and slight in 2. Peripheral oedema was usual but was often slight in comparison to the ascites and frequently disappeared on diuretic therapy when the ascites was resistant to treatment. The protein content of the ascitic fluid in 17 patients varied from $1.0 \mathrm{~g} . / 100 \mathrm{ml}$. to $6.0 \mathrm{~g} . / 100 \mathrm{ml}$., with a mean value of $3.5 \mathrm{~g} . / 100 \mathrm{ml}$. The cells were mostly lymphocytes and mesothelial cells. Uniformly blood-stained ascitic fluid was occasionally found, presumably due to spontaneous haemorrhage into the fluid. Chylous ascites has been seen once. Splenomegaly was common and in 9 patients exceeded 3 fingers' breadth, suggesting a cause other than cardiac failure; chronic malaria was probably responsible in most of them. Liver biopsies in 2 patients showed the histology of big spleen disease (Marsden et al., 1965). The high incidence of facial oedema was striking. Central cyanosis was not uncommon but was usually slight. There were no examples of marked clubbing but early clubbing occurred in 3 patients. Lid retraction and exophthalmos has been noticed in some patients.

TABLE II

PHYSICAL SIGNS OUTSIDE HEART IN 28 PATIENTS WITH ENDOMYOCARDIAL FIBROSIS

\begin{tabular}{l|c}
\hline \multicolumn{1}{c|}{ Physical signs } & $\begin{array}{c}\text { No. of } \\
\text { patients }\end{array}$ \\
\cline { 2 - 2 } Raised jugular venous pressure & 28 \\
Enlarged liver & 28 \\
Ascites & 28 \\
Peripheral oedema & 23 \\
Splenomegaly & 19 \\
Facial oedema & 15 \\
Central cyanosis & 10 \\
Dyspnoea & 6 \\
Early clubbing of digits & 3 \\
\hline
\end{tabular}

The systolic blood pressure was less than 100 $\mathrm{mm} . \mathrm{Hg}$ in 14 patients, between 100 and $120 \mathrm{~mm}$. $\mathrm{Hg}$ in 10 patients, and over $120 \mathrm{~mm}$. $\mathrm{Hg}$ in only 4 patients. At the time of presentation 7 patients had atrial fibrillation, all the others being in sinus rhythm. Three of the latter subsequently developed atrial fibrillation or flutter and in one of these the arrhythmia precipitated collapse and death. Table III shows the incidence of various signs in the heart.

In 5 patients the apex beat could not be localized and was difficult to feel in most of the others. Frequently there was only moderate or slight cardiac enlargement, as judged by the position of the apex beat. Pulsation was commonly seen in the left 2nd, 3rd, and 4th spaces, with a slight lift over it in a few instances. Contraction of the right ventricular infundibulum, which appears vigorous on cineangiography even when the right ventricular apex is obliterated by fibrosis, appears to account for this sign. A third sound is usual and is loudest over the right ventricle and its outflow tract. Pansystolic murmurs of grade $2 / 4$ or louder were heard in the mitral and tricuspid areas in 4 patients, and in the mitral area only in a further 4 patients. Twenty patients had no loud or sustained murmurs but in some of these very soft short systolic murmurs were sometimes heard at the apex or tricuspid area. 


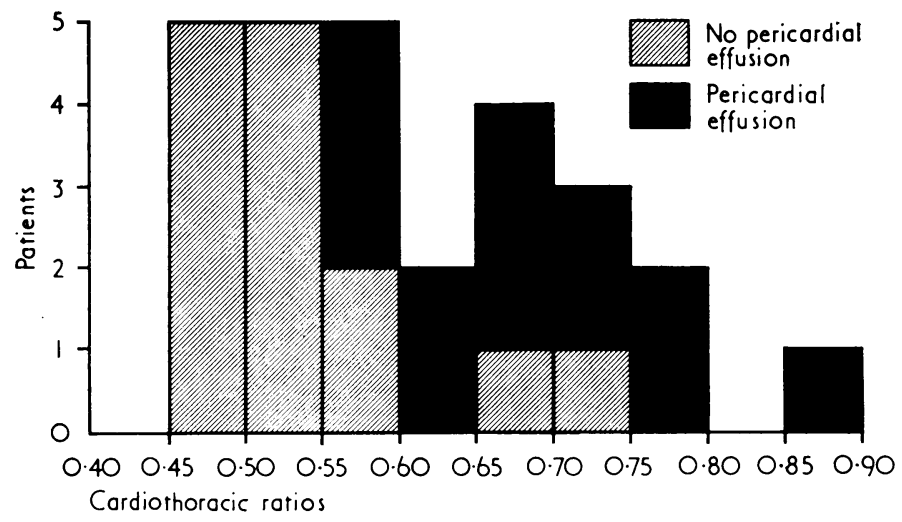

FIG. 3.-Cardiothoracic ratios in 27 patients with endomyocardial fibrosis.

Diastolic inflow murmurs following the third sound have been audible in some patients at the tricuspid or mitral area.

Radiological Appearance of Heart and Lungs. Fig. 3 illustrates the values for the cardiothoracic ratios in 27 patients. In one patient a large right pleural effusion made measurement impossible. In 10 patients with ratios below 0.55 there was only slight cardiac enlargement, but in many of these early dilatation of the right atrium was evident and there was a rounded contour to the left heart border instead of the normal concavity between the aortic

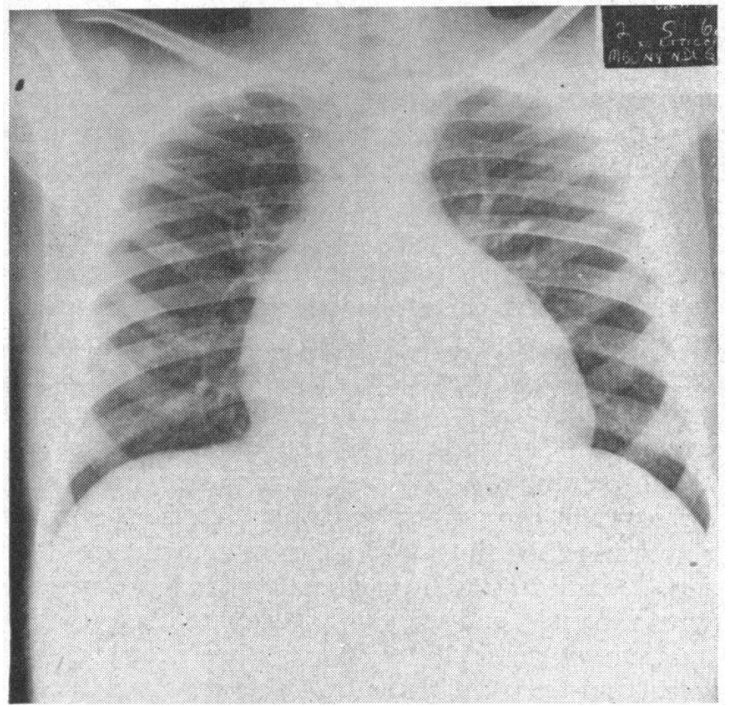

FIG. 4.-Chest radiograph showing moderate enlargement of the heart in a 14-year-old boy with endomyocardial fibrosis. Dilatation of the right atrium and of the infundibulum of the right ventricle give a rounded contour to both borders of the heart. knuckle and left ventricle. The appearances are illustrated in Fig. 4. Dilatation of the infundibulum of the right ventricle may account for the convexity of the left heart border, and cine-angiography seems to support this. In the left lateral view the retrosternal translucency is reduced in size as the right ventricular outflow tract encroaches upon it. The right and left pulmonary arteries appear normal or small. Moderate or gross degrees of cardiac enlargement are due either to more marked degrees of enlargement of the right atrium or to pericardial effusions or to a combination of these. Pericardial effusions were found in 13 patients and an effusion was present in all patients with a cardiothoracic ratio of 0.55 or greater, except in 3 patients whose marked cardiac enlargement was entirely due to gross right atrial dilatation. Endocardial calcification has been described in the right ventricle (Somers and Williams, 1962; Abrahams, 1962) but was only seen in 2 patients in this series. Both of

TABLE III

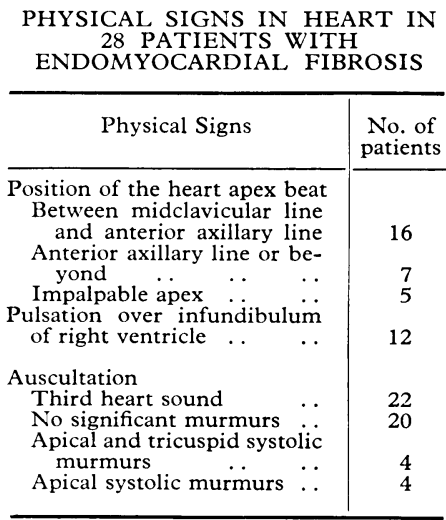



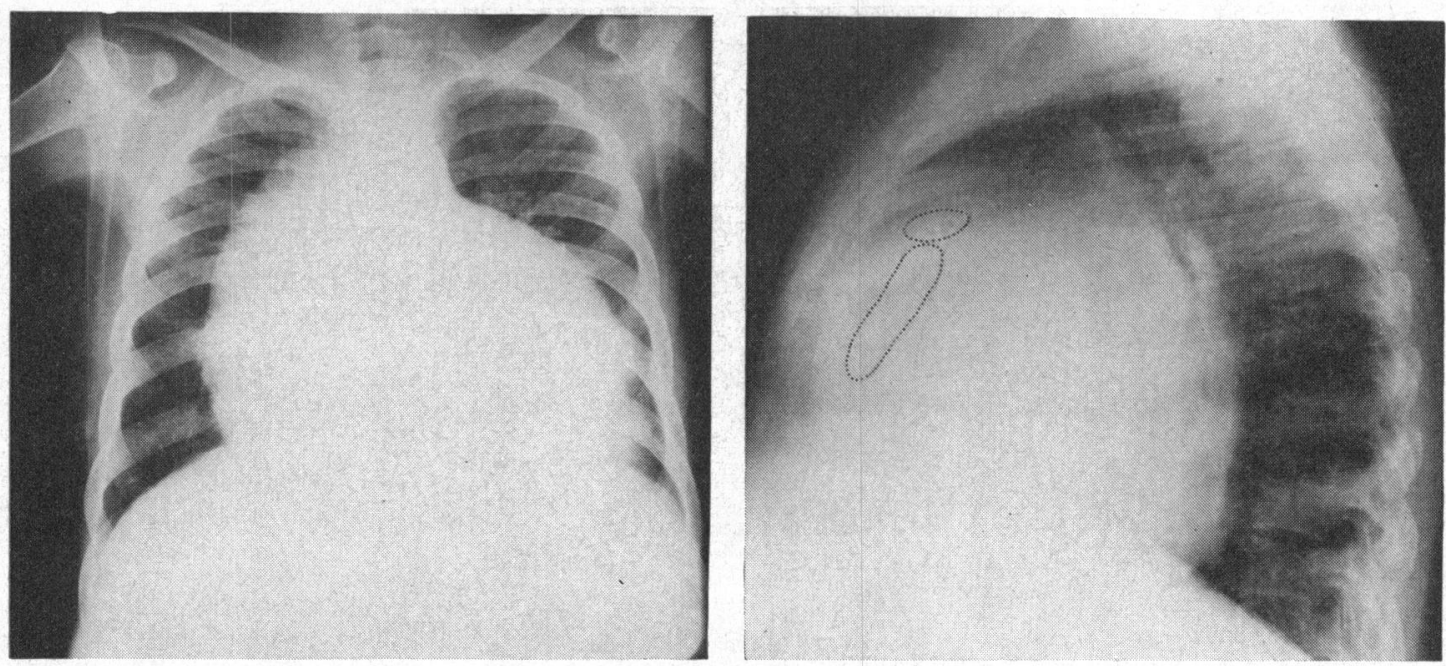

FIG. 5.-Chest radiographs from a patient with endomyocardial fibrosis (Case 3-see text). There is a giant right atrium. Pericardial effusion was excluded by coiling the catheter in the right atrium. Linear streaks of calcification (see lateral view) are probably in the endocardium of the right ventricle.

them had giant right atria (Fig. 5). Possible endocardial calcification was present in a further 2 patients but tomographic confirmation was not obtained.

Thirteen patients had pleural effusions and in 2 of them the effusions were Iarge, obscuring much of the right side of the chest. In 3 patients the effusion was associated with an underlying pathology which might have been pneumonia or pulmonary embolism. Very small right-sided pleural effusions were present in 6 of the 13 patients and small bilateral pleural effusions in a further 2 . Evidence of pulmonary congestion was present in only 2 patients. Their mean pulmonary artery pressures were the highest in the 26 patients $(40 \mathrm{~mm} . \mathrm{Hg}$ and $31 \mathrm{~mm}$. $\mathrm{Hg}$ ), and mitral systolic murmurs were also present, indicating coexistent left ventricular endomyocardial fibrosis.

Pericardial Effusion. Thirteen patients had a pericardial effusion, which was tapped in 11 of them, the volumes obtained ranging from $100-1000 \mathrm{ml}$. Air injections and radiography after removing the fluid always showed a thin pericardium and often a heart which was enlarged (Fig. 6). The fluid removed was usually clear yellow but sometimes blood stained, perhaps due to the trauma of the paracentesis. The protein content was commonly higher than in a simple transudate and a clot often formed in the removed fluid. The protein content of unclotted specimens from 10 patients varied from $2.4-7.0 \mathrm{~g}$. $/ 100 \mathrm{ml}$., with a mean of $4.2 \mathrm{~g} . / 100 \mathrm{ml}$. The fluid contained from a very few cells to several hundred per cu. mm. Usually they were lymphocytes and mesothelial cells. In some patients the effusions were chronic and have persisted for 3 years in 3 patients, 2 years in 1 patient, and 1 year in 2 patients.

Electrocardiographic Findings. Standard 12-lead electrocardiograms were available for analysis from all patients. Atrial fibrillation was found in 7 patients at the time of presentation and later atrial fibrillation or flutter developed in 3 others. Two patients presenting with atrial fibrillation subsequently had episodes of regular rhythm. Voltages were strikingly low in standard limb and unipolar leads when they were first seen but sometimes improved during follow-up. Pericardial effusion may have accounted for the low voltages in some patients but could not have done so in all of them. The mean QRS vector was usually normal and the heart vertical, semivertical, or intermediate in position. A left axis or horizontal heart position was uncommon. The corrected Q-T interval was normal in 80 per cent of patients when they were first seen.

An analysis of the $P$ wave was possible in 23 patients, and abnormalities were found in 11 of them either at the time of presentation or subsequently. An abnormally wide $P$ wave was the common finding and it was usually notched ( $P$ mitrale). In 3 of the 11 patients the $P$ wave exceeded $2 \mathrm{~mm}$. in lead V1 ( $P$ pulmonale). Atrioventricular conduction as judged by the duration of the $P-R$ interval was normal in most cases, being transiently prolonged to $\mathbf{0 . 2 2} \mathrm{sec}$. or greater in only 

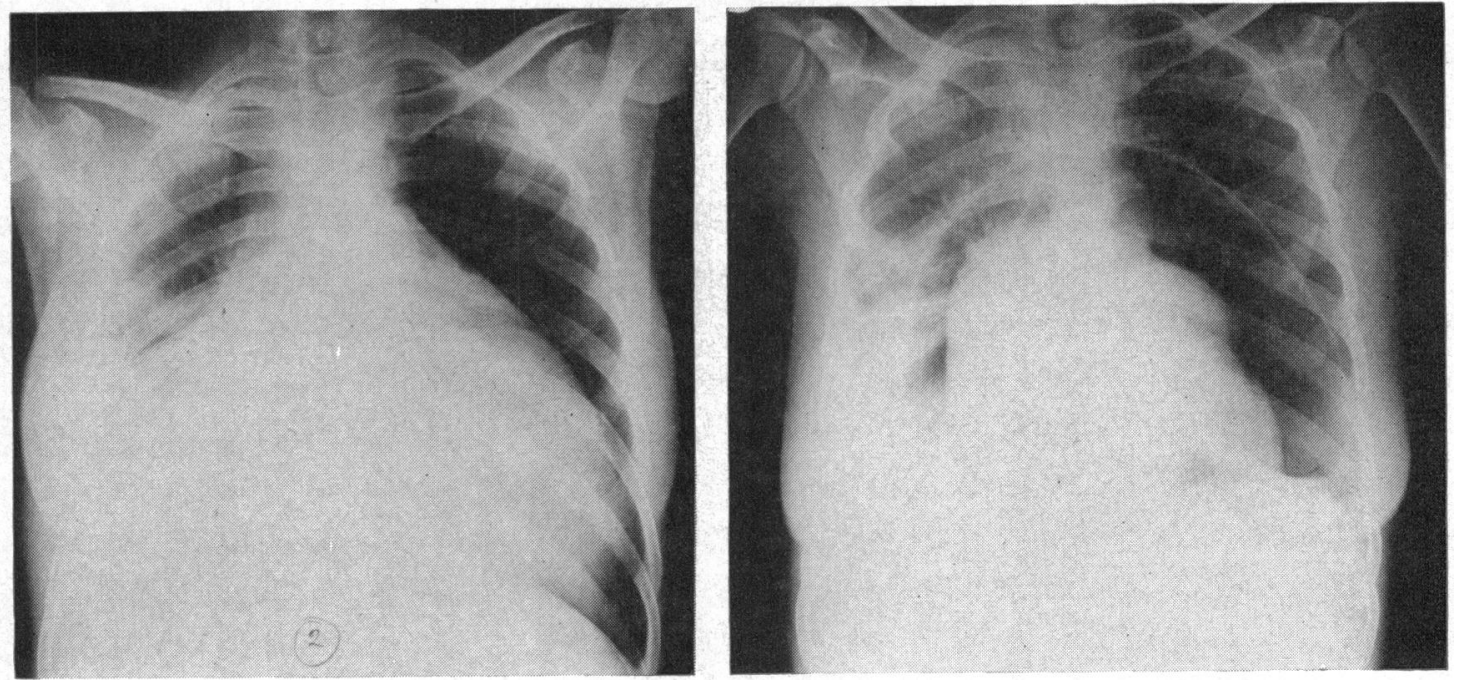

FIG. 6.-Chest radiographs from a patient with endomyocardial fibrosis (Case 1 - see text). There is a huge pericardial effusion and a large right pleural effusion. A normal thin pericardium is revealed after pericardial tapping. The right atrium and the infundibulum of the right ventricle appear large.

4 patients. Only one patient when first seen showed marked abnormality of intraventricular conduction, with a fully developed right bundle-branch block. This abnormality subsequently developed in a second patient after presentation. Three other patients have shown an $\mathrm{rsr}^{1}$ configuration in lead $\mathrm{V} 1$, with a duration of $0 \cdot 10-0 \cdot 12 \mathrm{sec}$. In addition to these, 10 patients had an rsr $^{1}$ configuration in lead V1 less than $0 \cdot 10 \mathrm{sec}$. long. These $\mathrm{rsr}^{1}$ complexes were usually of very low voltage, with all three waves approximately equal in size.

A dominant $R$ wave in AVR or a dominant $S$ in V5 occurred in 5 patients, suggesting right ventricular hypertrophy, but none exceeded a grade II right ventricular hypertrophy according to the criteria of Hollister and Goodwin (1963). T wave inversion in the right-sided chest leads extending to include V3 or even further was found in about half the patients. It was not infrequently associated with S-T segment depression in the right and midpraecordial-leads and elevation of the $S-T$ segment in AVR, a pattern reminiscent of subendocardial infarction. Sometimes this pattern developed after presentation (Fig. 7).

\section{DiAgNosis OF RIGHT VENTRICULAR ENDOMYOCARDIAL FIBROSIS}

When endomyocardial fibrosis affects only the right ventricle there appear to be three typical presentations if the clinical features are considered together with the appearance of the heart on the chest $x$-ray film. The three presentations are described below with illustrative case histories and their relative frequencies are represented in Fig. 8. One patient has been excluded from this classification because in her case there was obvious mitral incompetence with left atrial enlargement as a result of left ventricular fibrosis.

Patients with Large Pericardial Effusion and Cardiac Failure. The important differential diagnosis in this group of patients is from cardiac tamponade due to a pericardial effusion of tuberculous, pyogenic, or other aetiology. Thirteen of our patients had pericardial effusions, but in 3 of them the effusion was small and the radiographic appearance of the heart did not suggest its presence. These 3 are therefore excluded from this category and included in the others below. In 6 of the other 10 patients the possibility was raised of a cardiac abnormality additional to that of pericardial effusion by the presence of some of the features of tricuspid incompetence or of mitral systolic murmurs. In the remaining 4 patients all the clinical features could have been explained on the basis of pericarditis with effusion and cardiac tamponade. The characteristics of the pericardial fluid in endomyocardial fibrosis have been described above. Tapping the pericardial fluid may reveal a cardiac shadow with a large right atrium and prominent infundibulum suggestive of endomyocardial fibrosis. The pericardium appears thin (Fig. 6). The presence of an underlying primary cardiac disorder becomes obvious if the signs of cardiac failure persist after 


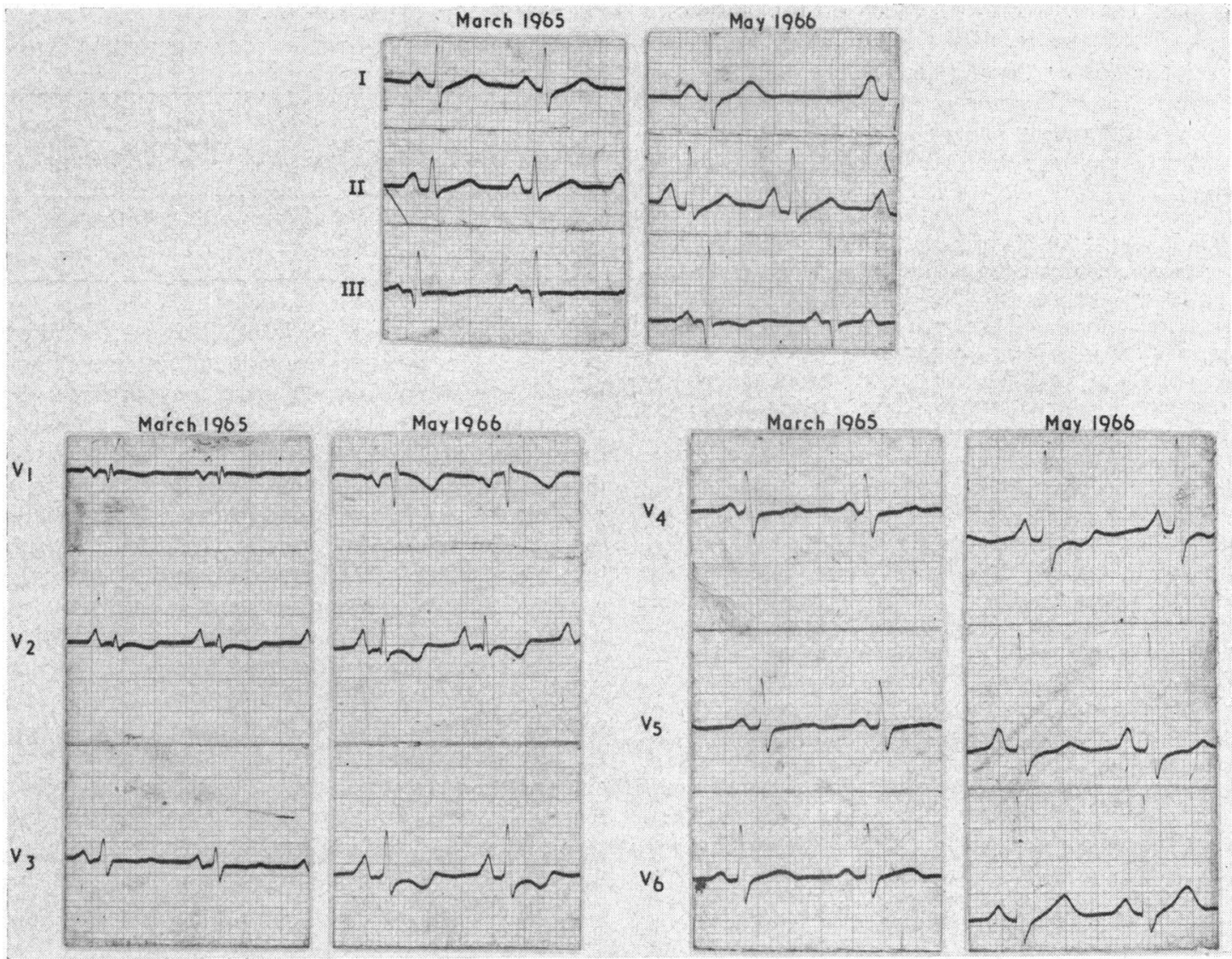

Fig. 7.-Electrocardiograms from a patient with endomyocardial fibrosis. The $P$ wave is tall and notched. $T$ wave inversion and $S-T$ segment depression in the right-sided chest leads have progressed between March 1965 and May 1966.

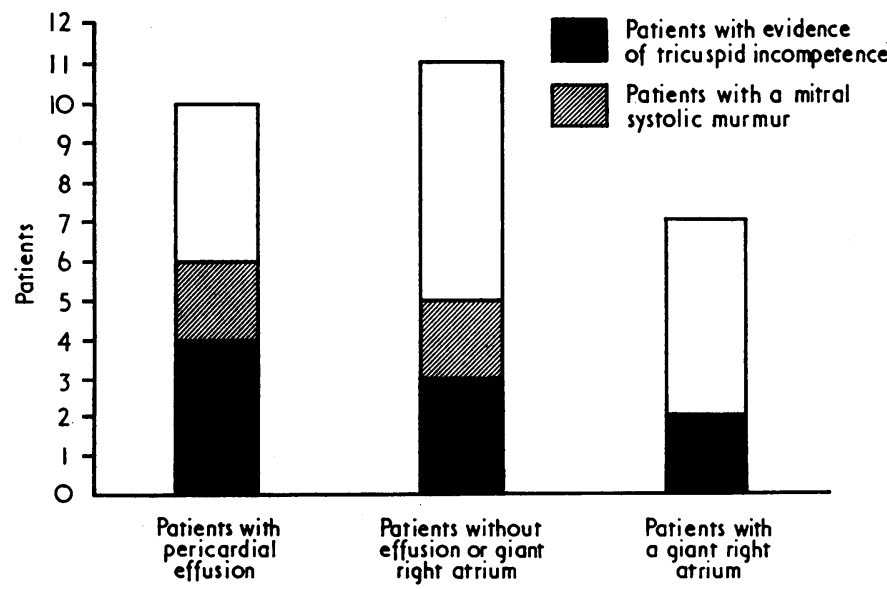

FIG. 8.-Patterns of presentation in 27 patients with severe ventricular endomyocardial fibrosis. 
tapping the effusion for relief of the suspected cardiac tamponade. In this situation endomyocardial fibrosis with pericardial effusion becomes the most likely diagnosis unless there is other evidence to suggest an acute rheumatic carditis. The diagnosis may be confirmed by cardiac catheterization.

Case 1. A 30-35-year-old Ganda woman gave a two-month history of headaches, palpitation, and breathlessness on exertion, with cough and abdominal swelling for a similar time. There was no haemoptysis. Swelling of both legs had been present for one month and facial swelling for 3 days. On examination she was an extremely ill woman with moderate central cyanosis, a heart rate of $120 / \mathrm{min}$., and a very paradoxical pulse. The systolic blood pressure was about $100 \mathrm{~mm}$. Hg. There was oedema of both feet and legs, marked facial oedema, and gross ascites. The jugular venous pressure was raised $20 \mathrm{~cm}$. to the ear lobes and the liver was enlarged 3-4 fingers' breadth. The cardiac apex was impalpable and the heart sounds very quiet. There were no murmurs. The electrocardiogram showed atrial fibrillation with low voltage in all leads. Lead V had an $\mathrm{rsr}^{1}$ configuration less than $0.08 \mathrm{sec}$. in duration, $r^{1}$ being the dominant wave. The chest $x$-ray revealed an enormous cardiac shadow strongly suggestive of a pericardial effusion (Fig. 6). Three hundred $\mathrm{ml}$. of fluid were removed from the pericardial sac, with some improvement in the patient's condition. The fluid was sterile on routine culture and contained protein $3 \mathrm{~g}$./100 $\mathrm{ml}$. and 175 cells/cu.mm., mostly lymphocytes. Tubercle bacilli were not seen or cultured. Over the next two weeks the pericardial sac was tapped on three occasions, with the removal of 800,600 , and $1000 \mathrm{ml}$. of fluid. The pulsus paradoxus became less pronounced and the pulse volume improved. However, the jugular venous pressure remained greatly raised, and after the injection of air into the pericardial sac, the pericardium was seen to be thin and the heart abnormal in shape, with a large right atrium and dilated infundibulum (Fig. 6). Endomyocardial fibrosis was suspected and confirmed by right heart catheterization and angiocardiography of the right ventricle. Pressures in the right side of the heart are recorded in Table IV.

Patients with Normal-sized or Moderately Enlarged Hearts and Cardiac Failure. The radiographic appearances of the heart in these patients do not suggest either pericardial effusion or giant right atria. However, some degree of right atrial enlargement is always present and can be proved by angiocardiography. This procedure may also reveal a small unsuspected pericardial effusion. Eleven patients have been included in this category, two of whom had very small pericardial effusions. The important differential diagnosis is from chronic and subacute constrictive pericarditis. Five of the 10 patients had either a mitral systolic murmur or evidence of trisuspid incompetence suggesting a
TABLE IV

PRESSURES (MM. HG) AT CARDIAC CATHETERIZATION IN 3 PATIENTS WITH ENDOMYOCARDIAL FIBROSIS

\begin{tabular}{|c|c|c|c|c|c|}
\hline & \multirow{2}{*}{$\begin{array}{c}\text { Right } \\
\text { atrium } \\
\text { mean }\end{array}$} & \multicolumn{2}{|c|}{ Right ventricle } & \multirow{2}{*}{$\begin{array}{c}\text { Pulmonary } \\
\text { artery } \\
\text { mean }\end{array}$} & \multirow{2}{*}{$\begin{array}{c}\text { Pulmonary } \\
\text { capillary } \\
\text { mean }\end{array}$} \\
\hline & & $\begin{array}{c}\text { End } \\
\text { diastolic }\end{array}$ & Systolic & & \\
\hline $\begin{array}{l}\text { Case } 2 \\
\text { Case } 1 \\
\text { Case } 3\end{array}$ & $\begin{array}{l}14 \\
22 \\
36\end{array}$ & $\begin{array}{l}13 \\
21 \\
27\end{array}$ & $\begin{array}{l}18 \\
31 \\
37\end{array}$ & $\begin{array}{l}16 \\
24 \\
34\end{array}$ & - \\
\hline
\end{tabular}

diagnosis other than pericardial disease. When evidence of a valvular lesion is absent, the radiological appearances of the heart may provide the only other help in diagnosis. Pericardial calcification almost excludes endomyocardial fibrosis as the diagnosis and has only once been seen by us in a possible example of this disease. Conversely, calcification in the wall of the ventricle does not occur in chronic constrictive pericarditis. It only occurred in 2 of our 28 patients with endomyocardial fibrosis but is a very useful diagnostic aid when present. In patients without evidence of a valvular lesion or calcification in the pericardium or ventricular wall the differential diagnosis is difficult. All the characteristic clinical features of chronic constrictive pericardial disease can occur in endomyocardial fibrosis of the right ventricle, including the paradoxical arterial pulse, the sharp $y$ descent of the venous pulse, the quiet heart apex beat, and the early third sound.

Case 2. A 26-year-old Rwanda man of the Hutu tribe had lived in Uganda for 10 years. His illness began in 1964, with swelling of the legs, face, and abdomen. He was admitted to Mulago Hospital 2 years later when he also complained of palpitations, breathlessness on exertion, and cough. There was no history of haemoptysis, orthopnoea, or paroxysmal nocturnal dyspnoea. On examination the patient was in regular rhythm, with a slightly paradoxical pulse of small volume. There was peripheral cyanosis and the blood pressure was 110/80 $\mathrm{mm}$. Hg. The jugular venous pressure was raised 15 $\mathrm{cm}$., with a normal venous wave pattern, and there was oedema of the legs and face, with a moderate ascites. The apex beat of the heart was impalpable. There were no murmurs, but a soft 3 rd sound was audible at the left sternal edge. The electrocardiogram was generally of very low voltage with a P mitrale in lead II but was otherwise quite normal. The radiological appearance of the heart (Fig. 9) did not suggest any particular form of heart disease. There was no pericardial or endocardial calcification. The most likely diagnoses were thought to be constrictive pericarditis or endomyocardial fibrosis. Right heart catheterization and cine-angiography of the right ventricle were performed (Fig. 10). The pressures are given in Table IV. Both pressure tracings and cine-angiography were characteristic of right ventricular endomyocardial fibrosis. 
Patients with a Giant Right Atrium and Cardiac Failure. Six of our patients have been included in this group and in two of these there was evidence of tricuspid incompetence. The differential diagnosis is from other causes of tricuspid incompetence or giant right atrium. Tricuspid incompetence without other valvular lesions is extremely uncommon in rheumatic heart disease. Isolated tricuspid stenosis may result in a giant right atrium, but it is rare and characterized by a large $a$ wave in the venous pulse and a diastolic murmur. A large right atrium and tricuspid incompetence occur in Ebstein's anomaly which is also a rare cardiac disorder. The diagnostic problem in this group of patients is to be sure that the abnormal cardiac configuration is really due to a large right atrium. A moderately large right atrium may give a rounded contour to the appearance of the heart not altogether unlike that of a pericardial effusion.

Case 3. A 14-year-old girl (Fig. 11) gave a history of swelling of the abdomen for one year, followed by swelling of the face, dyspnoea, and palpitations on exertion, and a dry cough. On examination there was early clubbing, slight peripheral oedema, and a very gross ascites. There was atrial fibrillation with a slow ventricular rate before any digitalis preparation was given. The blood pressure was $120 / 90 \mathrm{~mm}$. $\mathrm{Hg}$, and the jugular venous pressure was raised to the ears. The apex beat of the heart was quiet and it was slightly displaced to the left. The heart sounds were normal and there were no murmurs. The electrocardiogram showed atrial fibrillation, right axis deviation, and a vertical heart position. There was a dominant $R$ wave in $V 1$ and a dominant $S$ in V5 with $\mathrm{T}$ wave inversion from V1-V4. The radiological appearance of the heart suggested a giant right atrium. Calcification was seen which appeared to be in the right ventricular wall (Fig. 5). Severe right ven-

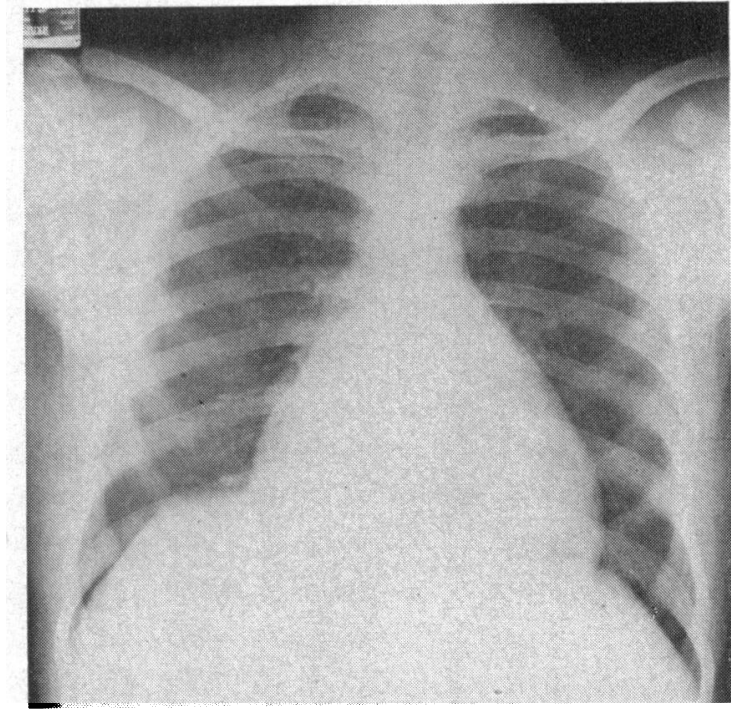

Fig. 9. - Chest radiograph from a patient with endomyocardial fibrosis (Case 2-see text). There was no particular effusion. The right atrium is not obviously enlarged.

tricular endomyocardial fibrosis was diagnosed. The pressures at right heart catheterization are recorded in Table IV.

When endomyocardial fibrosis affects the left ventricle to a significant degree the differential diagnosis becomes wider and much more difficult. The signs of right-sided heart failure may then be interpreted as secondary to a left-sided heart lesion. If the endomycocardial fibrosis has damaged the mitral valve, a diagnosis of rheumatic heart disease may be made. If mitral murmurs are absent but
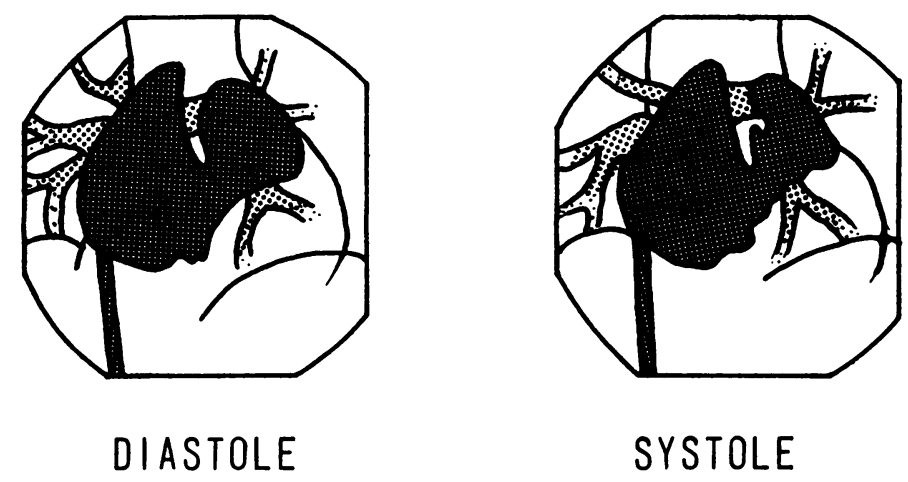

FIG. 10.-Line diagrams traced from a $16 \mathrm{~mm}$. cine-angiography film obtained from a patient with endomyocardial fibrosis (Case $2-$ see text). The catheter lies in the inferior vena cava with its tip in the right atrium. There is no pericardial effusion. The right atrium is large. The apex of the right ventricle is absent and the body of the right ventricle is constricted, but the infundibulum is dilated. 


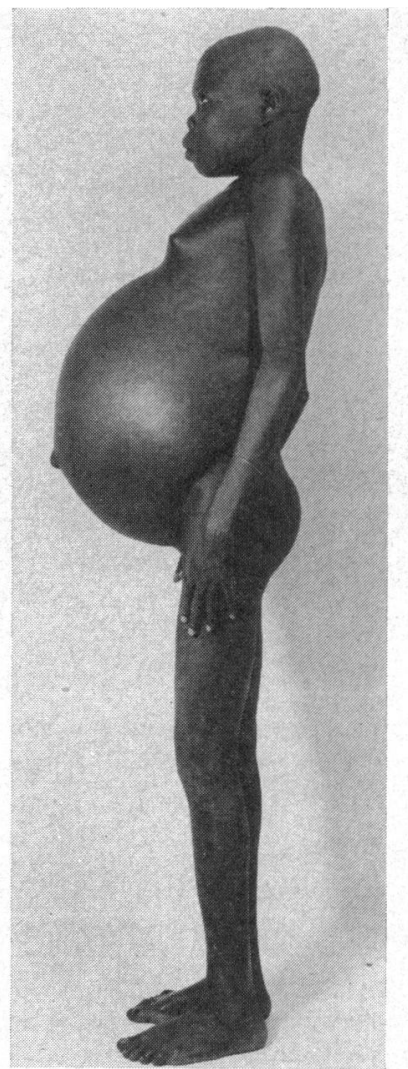

FIG. 11.-A young girl with severe right ventricular endomyocardial fibrosis (Case 3-see text). Note the huge ascites and the absence of gross ankle oedema.

pulmonary congestion indicates a left-sided lesion then congestive cardiomyopathy enters the differential diagnosis (Goodwin et al., 1961). Neither rheumatic heart disease nor congestive cardiomyopathy need be considered if it is clear that symptoms and signs of pulmonary congestion are absent.

\section{Diagnostic Value of Right Heart CATHETERIZATION}

When the left ventricle is unaffected in endomyocardial fibrosis the pulmonary capillary and pulmonary artery pressures are normal. The fibrotic process in the right ventricle reduces its compliance, and the end-diastolic pressure becomes raised with a corresponding rise in the right atrial pressure. Ventricular systole may fail to raise the right ventricular pressure more than a few $\mathrm{mm}$. Hg above the end-diastolic pressure. The tricuspid valve is usually incompetent and so atrial and ventricular pressures may be almost equal during ventricular systole. In this way the configuration of the atrial and ventricular pressure tracings becomes almost identical.

The mean pressures in right atrium, right ventricle, and pulmonary artery approximate to each other. In patients with constrictive pericarditis the pulmonary capillary and artery pressures are usually both slightly raised because the left ventricle shares in the constriction. This is sometimes a helpful point in differentiating right ventricular endomyocardial fibrosis from constrictive pericarditis (Fig. 12).

Table IV gives the values for the right-sided heart pressures in the 3 patients whose histories are described above.

The pressures in Case 2 are typically those of right ventricular endomyocardial fibrosis, as described above. A satisfactory measurement of the pulmonary capillary pressure was not obtained in either of the other 2 patients. The pressures measured in Case 1 strongly suggest endomyocardial fibrosis, however, because of the high end-diastolic pressure in the ventricle in the presence of a mean pulmonary artery pressure at the upper limit of normal. Moderate pulmonary hypertension was present in Case 3 but again the end-diastolic pressure in the ventricle was extremely high. In this patient a giant right atrium helped to exclude constrictive pericarditis as a diagnosis.

The angiocardiographic appearances of endomyocardial fibrosis have been described by Cockshott (1965). In the right ventricular form of the disease the body of the ventricle appears small and the apex absent. The right atrium is large and there is free tricuspid regurgitation. The appearances are illustrated in Fig. 10 from Case 2. The procedure is of most value when the pressure tracings alone are not diagnostic. This is the situation when right ventricular endomyocardial fibrosis is combined with sufficient left ventricular disease to cause an increase in the pulmonary pressures.

\section{Discussion}

Clinical Features. Two-thirds of the patients reported in this series were from the Rwanda tribes. The susceptibility of these tribes to the disease had been previously well established by others from clinical and necropsy studies. It remains to be established whether this susceptibility depends upon genetic or environmental factors, but a number of our patients had lived in Uganda for several years before the onset of symptoms.

The patients reported here were seen in adult clinics and wards, but most were under 15 years old at the onset of the disease. The illness may begin in many patients under 10 years of age, with consequent admission to the paediatric wards where we 


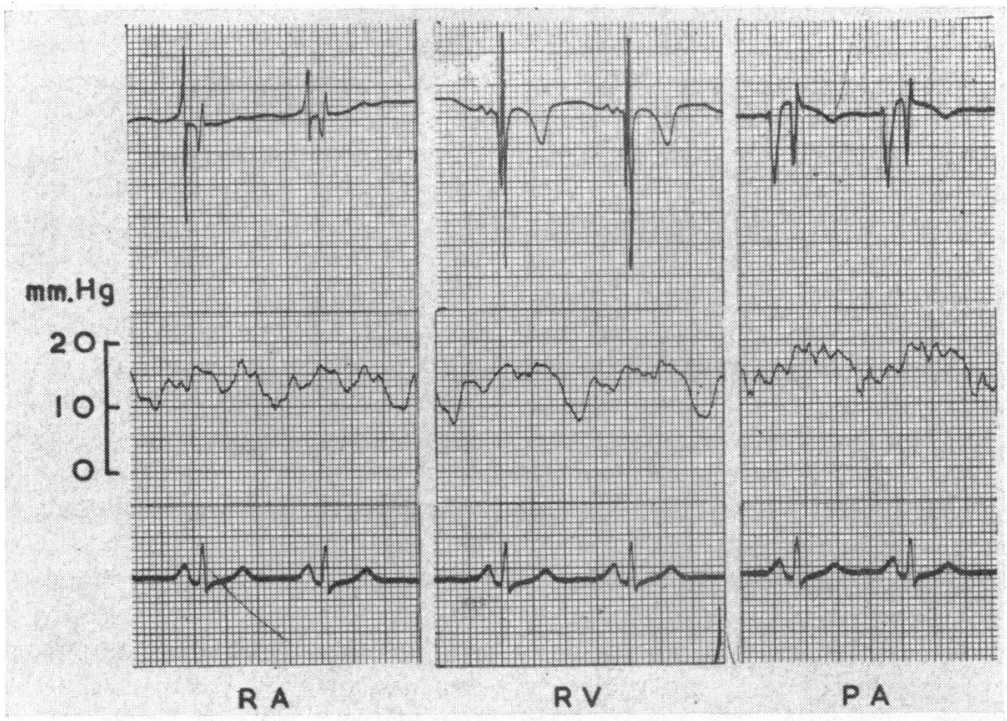

Fig. 12.-Pressure records from right atrium (RA), right ventricle (RV), and pulmonary artery (PA) recorded in a patient with endomyocardial fibrosis (Case 2-see text). The intracardiac electrogram is at the top and standard lead II at the bottom.

may not have seen them. The commonest cardiac diagnosis among the paediatric admissions to Mulago Hospital is carditis (Caddell et al., 1966), and sometimes the clinical evidence that it is rheumatic carditis is scanty. It seems likely that some of these patients are at an early stage in the natural history of endomyocardial fibrosis. Parry and Abrahams (1965) have described patients with an acute onset of fever, malaise, tachycardia, and cardiac failure, whose hearts show the typical fibrotic endocardial scars at necropsy together with the histological picture of an acute inflammatory reaction in the subendocardial layers. Similar cases following a rapid downhill course to death have been seen here, but it is our experience that patients presenting in the chronic state of the disease can rarely give an accurate account of exactly when and how their illness began. From the patients described in this paper it was not possible to establish the exact mode of onset of the disease. Fever frequently is used by patients to mean little more than malaise, and other general symptoms such as lassitude and weakness of which patients complain at the start of the illness may all be ascribed to a low cardiac output. All this serves to emphasize the need for a well-organized follow-up study of cases of acute heart failure in young children to see how many develop the recognizable features of established endomyocardial fibrosis in later life. Indeed, if there is any truth in the theory that endomyocardial fibrosis is a sequel to a streptococcal infection like rheumatic heart disease (Shaper, 1966), then the onset of these two conditions may be identical. A clinical study and follow-up of children with heart failure of uncertain aetiology would provide valuable evidence for or against the streptococcal theory and contribute important knowledge concerning the natural history of endomyocardial fibrosis.

The occurrence of a raised jugular venous pressure, hepatomegaly, oedema, and ascites in the absence of pulmonary congestion strongly suggests a diagnosis of severe right ventricular endomyocardial fibrosis. Marked disproportion between the large ascites and the slight or moderate peripheral oedema has been emphasized by Abrahams (1962). This disproportion is not peculiar to endomyocardial fibrosis, however, and is seen here in some cases of rheumatic heart disease also. Diuretic therapy may make the disproportion more obvious, since the peripheral oedema may disappear while the ascites persists. The protein content of the ascitic fluid in the patients reported has averaged $3.5 \mathrm{~g} . / 100 \mathrm{ml}$., a figure higher than that of most transudates. It is high in constrictive pericarditis, however, and values of over $3 \mathrm{~g} . / 100 \mathrm{ml}$. are quoted by Spodick (1964). Only further studies will show whether there is anything peculiar about the nature or formation of ascitic fluid in endomyocardial fibrosis. Similar considerations apply to facial oedema. It was noted in 15 of the patients in this series, but it is occasionally seen in severe right heart failure of other aetiology. The splenomegaly seen in many of our 
patients is probably also coincidental and not part of the disease process leading to endomyocardial fibrosis. Spleens weighing more than $300 \mathrm{~g}$. are found in the majority of Rwandans at necropsy, regardless of the cause of death. Malaria probably accounts for much of this splenomegaly.

Patients from Nigeria with typical severe right ventricular endomyocardial fibrosis have been described as having a heart made massive by an aneurysmal right atrium (Parry, 1964). In our experience a right atrium as large as that illustrated in Fig. 5, which is comparable to the pictures published from Nigeria (Abrahams, 1962), is not common and not representative of most patients with severe right ventricular disease. A lesser degree of right atrial enlargement is more usual, and a grossly enlarged heart shadow on the chest $x$-ray film is often due to a large pericardial effusion. The cause of the pericardial effusion is uncertain, but it may depend in part or in whole upon inflammatory reactions in the pericardial layers. This would also explain the high protein content of some of the effusions. The pathological process in endomyocardial fibrosis should be regarded as a pancarditis since all layers of the heart are involved.

Characteristically the fibrosis of the endocardium begins at the apex and extends upwards until the papillary muscles and chordae tendineae are involved in the process (see Fig. 1). Tricuspid incompetence is inevitable, and yet the characteristic murmur is often absent even when there is a prominent systolic wave in the jugular pulse and even systolic expansion of the liver. This may be because the tricuspid valve ring is so dilated in many patients that little turbulence is set up during the systolic regurgitation. Abrahams (1962) reported that systolic expansion of the neck veins is the rule in right ventricular endomyocardial fibrosis. For patients in sinus rhythm it has been our experience that the $a$ and $v$ waves of the venous pulse are usually equal in size and the $x$ and $y$ descents prominent. Right atrial pressure tracings reveal that often both the $a$ and $v$ waves are large, indicating a strong atrial contraction and tricuspid regurgitation. The incompetence of the tricuspid valve is also indicated by the finding that in most patients there is little or no pressure gradient between the atrium and right ventricle during ventricular systole. A third sound, loudest over the right ventricle or its outflow tract, is one of the commonest auscultatory features of the disease. It may be high in pitch and unusually early, rather like the early diastolic sound of chronic constrictive pericarditis (Mounsey, 1955).

The chief electrocardiographic features of severe endomyocardial fibrosis of the right ventricle have been described by others (Abrahams, 1962; Williams and Somers, 1960). Attention has been drawn in these publications to the low voltages and the frequent occurrence of a $P$ mitrale. These were also common findings in our present series of patients. $T$ wave inversion spreading across the chest leads from V1 to V3 or further, was not uncommon. This is probably a significant abnormality particularly if associated with S-T segment depression. $T$ wave inversion or biphasic $T$ waves from V1 to V3 or further, were recorded in about 15 per cent of normal university students of Bantu origin by Somers and Rankin (1962), but was usually associated with an increase in the S-T segment. The combination of $T$ wave inversion and S-T segment depression is like that of subendocardial infarction, and could be attributed to the endocardial and subendocardial changes in endomyocardial fibrosis. The same pattern may be seen in association with marked right ventricular hypertrophy, but none of our patients had electrocardiographic evidence of severe hypertrophy. Some patients had evidence of grade I hypertrophy (Hollister and Goodwin, 1963). It is not easy to understand why there should be any evidence of right ventricular hypertrophy since the right ventricular apex is fibrosed and shrunken. However, the right ventricular outflow tract is dilated and hypertrophied, and perhaps this is one explanation. Alternatively, a dominant $S$ wave in V5 may be attributed to clockwise rotation of the heart by an enlarged right atrium rather than to right ventricular hypertrophy. Abrahams (1962) considered that $T$ wave inversion from V1-V4, which occurred in most of his patients, was also due to cardiac rotation. Clockwise rotation, however, would not explain the S-T segment depression in these leads. The low voltage rsr $^{1}$ pattern commonly seen in lead V1, in the absence of other criteria of complete right bundle-branch block, is presumably due to abnormal rates or pathways of right ventricular excitation.

\section{SUMMARY}

The clinical features of 28 patients with endomyocardial fibrosis affecting predominantly the right ventricle are described. One-third of patients were under 15 years of age. Pericardial effusions were found in 13 patients. The clinical signs of tricuspid incompetence were found in less than half the patients. A large right atrium was not always evident on routine postero-anterior and lateral radiographs of the chest but was invariably found on cine-angiography. Typical case presentations are discussed with particular reference to diagnosis and the value of right heart catheterization and cineangiography. 
We are grateful to Professor J. F. Goodwin for his helpful advice and to Professor J. A. Tulloch for his encouragement. Dr. P. G. D'Arbela, Dr. B. R. Kanyerezi, and Dr. J. R. Billinghurst have all contributed to the clinical observations. Mr. B. J. Milton and $\mathrm{Mr}$. D. Havey have rendered skilled and invaluable technical assistance. Mr. W. Serumaga kindly drew the illustrations, and the photographs were supplied by Mr. J. E. Busulwa.

Generous research grants from the Medical Research Council (London) and National and Grindlays Bank (Uganda) to one of us (K. Somers) financed the purchase of the equipment of the cardiac catheter laboratory. We have also received capital and recurrent support from the British Heart Foundation (1966 and 1967). Mr. E. J. Baines (May \& Baker Ltd., Dagenham, England) has supplied us with generous gifts of contrast medium for angiocardiography.

\section{REFERENCES}

Abrahams, D. G. (1962). Endomyocardial fibrosis of the right ventricle. Quart. F. Med., 31, 1.

$\longrightarrow$, and Parry, E. H. O. (1963). Chronic pericardial effusion complicating endomyocardial fibrosis. Circulation, 28, 221.

Ball, J. D., Williams, A. W., and Davies, J. N. P. (1954). Endomyocardial fibrosis. Lancet, 1, 1049.

Caddell, J. L., Warley, A., Connor, D. H., D'Arbela, P. G., and Billinghurst, J. R. (1966). Acquired heart disease in Ugandan children. Brit. Heart f., 28, 759.

Cockshott, W. P. (1965). Angiocardiography of endomyocardial fibrosis. Brit. F. Radiol., 38, 192.

Connor D. H., Somers, K., Hutt, M. S. R., Manion, W. C., and D'Arbela, P. G. (1967). Endomyocardial fibrosis in Uganda (Davies' disease). Part I, an epidemiologic, clinical, and pathologic study. Amer. Heart f., 74, 687.

$\longrightarrow,-\frac{}{7 .}, 75,107$.

Davies, J. N. P. (1948). Endocardial fibrosis in Africans. E. Afr. med. F., 25, 10.

Goodwin, J. F., Gordon, H., Hollman, A., and Bishop, M. B. (1961). Clinical aspects of cardiomyopathy. Brit. med. f., 1, 69.
Hollister, R. M., and Goodwin, J. F. (1963). The electrocardiogram in cardiomyopathy. Brit. Heart f., 25, 357.

Marsden, P. D., Hutt, M. S. R., Wilks, N. E., Voller, A., Blackman, V., Shah, K. K., Connor, D. H., Hamilton, P. J. S., Banwell, J. G., and Lunn, H. F. (1965). An investigation of tropical splenomegaly at Mulago Hospital, Kampala, Uganda. Brit. med. F., 1, 89.

Mounsey, P. (1955). The early diastolic sound of constrictive pericarditis. Brit. Heart f., 17, 143.

Parry, E. H. O. (1964). Endomyocardial fibrosis. Ciba Foundation Symposium: Cardiomyopathies. Ed. by G. E. W. Wolstenholme and M. O'Connor, p. 322. Churchill, London.

- , and Abrahams, D. G. (1963). The function of the heart in endomyocardial fibrosis of the right ventricle. Brit. Heart F., 25, 619.

$\longrightarrow$, and - (1965). The natural history of endomyocardial fibrosis. Quart. F. Med., 34, 383.

Shaper, A. G. (1966). Endomyocardial fibrosis and rheumatic heart-disease. Lancet, 1, 639.

Shillingford, J. P., and Somers, K. (1961). Clinical and haemodynamic patterns in endomyocardial fibrosis. Brit. Heart F., 23, 433.

Somers, K., and D'Arbela, P. G. (1964). Heart diseases in Uganda. Proc. Third Asian-Pacific Congr. Cardiol, 1, 162.

- , and Rankin, A. M. (1962). The electrocardiogram in healthy East African (Bantu and Nilotic) men. Brit. Heart F., 24, 542.

—, and Williams, A. W. (1962). Intracardiac calcification in endomyocardial fibrosis. Brit. Heart $\mathcal{F}$., 24, 324.

Spodick, D. H. (1964). Chronic and Constrictive Pericarditis. Grune and Stratton, New York and London.

Van der Geld, H., Peetoom, F., Somers, K., and Kanyerezi, B. R. (1966). Immunohistological and serological studies in endomyocardial fibrosis, Lancet, 2, 1210.

Williams, A. W., Ball, J. D., and Davies, J. N. P. (1954). Endomyocardial fibrosis in Africa: its diagnosis, distribution and nature. Trans. roy. Soc. trop. Med. Hyg., 48, 290.

- and Somers, K. (1960). The electrocardiogram in endomyocardial fibrosis. Brit. Heart $\mathcal{f}$., 22, 311.

W.H.O. Memorandum (1965). Cardiomyopathies. Bull. Wld Hlth Org., 33, 257. 